\title{
Mappings of type generalized de La Vallée Poussin's mean
}

\section{Awad A Bakery*}

\section{"Correspondence:}

awad_bakery@yahoo.com;

awad_bakry@hotmail.com Department of Mathematics,

Faculty of Science and Arts, King Abdulaziz University (KAU), P.O. Box 80200, Khulais, Code 21589, Saudi Arabia

Department of Mathematics, Faculty of Science, Ain Shams University, Abbassia, P.O. Box 1156 , Cairo, 11566, Egypt

\begin{abstract}
In the present paper, we study the operator ideals generated by the approximation numbers and generalized de La Vallée Poussin's mean defined in (Şimşek et al. in J. Comput. Anal. Appl. 12(4):768-779, 2010). Our results coincide with those in (Faried and Bakery in J. Inequal. Appl. 2013, doi:10.1186/1029-242X-2013-186) for the generalized Cesáro sequence space.
\end{abstract}

Keywords: approximation numbers; operator ideal; generalized de La Vallée Poussin's mean sequence space

\section{Introduction}

By $L(X, Y)$ we denote the space of all bounded linear operators from a normed space $X$ into a normed space $Y$. The set of nonnegative integers is denoted by $\mathbb{N}=\{0,1,2, \ldots\}$ and the real numbers by $\mathbb{R}$. By $\omega$ we denote the space of all real sequences. A map which assigns to every operator $T \in L(X, Y)$ a unique sequence $\left(s_{n}(T)\right)_{n=0}^{\infty}$ is called an $s$-function and the number $s_{n}(T)$ is called the $n$th $s$-numbers of $T$ if the following conditions are satisfied:

(a) $\|T\|=s_{0}(T) \geq s_{1}(T) \geq \cdots \geq 0$ for all $T \in L(X, Y)$.

(b) $s_{n}\left(T_{1}+T_{2}\right) \leq s_{n}\left(T_{1}\right)+\left\|T_{2}\right\|$ for all $T_{1}, T_{2} \in L(X, Y)$.

(c) $s_{n}(R S T) \leq\|R\| s_{n}(S)\|T\|$ for all $T \in L\left(X_{0}, X\right), S \in L(X, Y)$ and $R \in L\left(Y, Y_{0}\right)$.

(d) $s_{n}(\lambda T)=|\lambda| s_{n}(T)$ for all $T \in L(X, Y), \lambda \in \mathbb{R}$.

(e) $\operatorname{rank}(T) \leq n$ if $s_{n}(T)=0$ for all $T \in L(X, Y)$.

(f)

$$
s_{r}\left(I_{n}\right)= \begin{cases}1 & \text { for } r<n, \\ 0 & \text { for } r \geq n,\end{cases}
$$

where $I_{n}$ is the identity operator on the Euclidean space $\ell_{2}^{n}$.

As examples of $s$-numbers, we mention approximation numbers $\alpha_{n}(T)$, Gelfand numbers $c_{n}(T)$, Kolmogorov numbers $d_{n}(T)$ and Tichomirov numbers $d_{n}^{*}(T)$ defined by:

(I) $\alpha_{n}(T)=\inf \{\|T-A\|: A \in L(X, Y)$ and $\operatorname{rank}(A) \leq n\}$.

(II) $c_{n}(T)=a_{n}\left(J_{Y} T\right)$, where $J_{Y}$ is a metric injection (a metric injection is a one-to-one operator with closed range and with norm equal to one) from the space $Y$ into a higher space $\ell^{\infty}(\Lambda)$ for a suitable index set $\Lambda$.

(III) $d_{n}(T)=\inf _{\operatorname{dim} Y \leq n} \sup _{\|x\| \leq 1} \inf _{y \in Y}\|T x-y\|$

(IV) $d_{n}^{*}(T)=d_{n}\left(J_{Y} T\right)$

o2013 Bakery; licensee Springer. This is an Open Access article distributed under the terms of the Creative Commons Attribution License (http://creativecommons.org/licenses/by/2.0), which permits unrestricted use, distribution, and reproduction in any medium, provided the original work is properly cited. 
All these numbers satisfy the following condition:

(g) $s_{n+m}\left(T_{1}+T_{2}\right) \leq s_{n}\left(T_{1}\right)+s_{m}\left(T_{2}\right)$ for all $T_{1}, T_{2} \in L(X, Y)$.

An operator ideal $U$ is a subclass of $L=\{L(X, Y) ; X$ and $Y$ are Banach spaces $\}$ such that its components $\{U(X, Y) ; X$ and $Y$ are Banach spaces $\}$ satisfy the following conditions:

(i) $I_{K} \in U$, where $K$ denotes the 1-dimensional Banach space, where $U \subset L$.

(ii) If $T_{1}, T_{2} \in U(X, Y)$, then $\lambda_{1} T_{1}+\lambda_{2} T_{2} \in U(X, Y)$ for any scalars $\lambda_{1}, \lambda_{2}$.

(iii) If $V \in L\left(X_{0}, X\right), T \in U(X, Y)$ and $R \in L\left(Y, Y_{0}\right)$, then $R T V \in U\left(X_{0}, Y_{0}\right)$. See $[1,2]$ and [3].

For a sequence $p=\left(p_{n}\right)$ of positive real numbers with $p_{n} \geq 1$, for all $n \in \mathbb{N}$, the generalized Cesáro sequence space is defined by

$$
\begin{aligned}
& \operatorname{Ces}(p)=\left\{x=\left(x_{k}\right) \in \omega: \rho(\lambda x)<\infty \text { for some } \lambda>0\right\}, \\
& \text { where } \rho(x)=\sum_{n=0}^{\infty}\left(\frac{1}{n+1} \sum_{k=0}^{n}\left|x_{k}\right|\right)^{p_{n}} .
\end{aligned}
$$

The space $\operatorname{Ces}(p)$ is a Banach space with the norm $\|x\|=\inf \left\{\lambda>0: \rho\left(\frac{x}{\lambda}\right) \leq 1\right\}$.

If $p=\left(p_{n}\right)$ is bounded, we can simply write $\operatorname{Ces}(p)=\left\{x \in \omega: \sum_{n=0}^{\infty}\left(\frac{1}{n+1} \sum_{k=0}^{n}\left|x_{k}\right|\right)^{p_{n}}<\infty\right\}$. Also, some geometric properties of $\operatorname{Ces}(p)$ are studied in [4-6] and [7].

Let $\Lambda=\left(\lambda_{n}\right)$ be a nondecreasing sequence of positive real numbers tending to infinity, and let $\lambda_{0}=1$ and $\lambda_{n+1} \leq \lambda_{n}+1$.

De La Vallée Poussin's means of a sequence $x=\left(x_{k}\right)$ are defined as follows:

$$
t_{n}(x)=\frac{1}{\lambda_{n}} \sum_{j \in I_{n}}\left|x_{j}\right|, \quad \text { where } I_{n}=\left[n-\lambda_{n}+1, n\right], \text { for } k \in \mathbb{N} .
$$

The generalized de La Vallée Poussin's mean sequence space was defined in [8].

$$
V(\lambda, p)=\{x \in \omega: \rho(\lambda x)<\infty \text { for some } \lambda>0\}, \quad \text { where } \rho(x)=\sum_{n=0}^{\infty}\left(\frac{1}{\lambda_{n}} \sum_{k \in I_{n}}\left|x_{k}\right|\right)^{p_{n}} .
$$

The space $V(\lambda, p)$ is a Banach space with the norm

$$
\|x\|=\inf \left\{\lambda>0: \rho\left(\frac{x}{\lambda}\right) \leq 1\right\} .
$$

If $p=\left(p_{n}\right)$ is bounded, we can simply write

$$
V(\lambda, p)=\left\{x \in \omega: \sum_{n=0}^{\infty}\left(\frac{1}{\lambda_{n}} \sum_{k \in I_{n}}\left|x_{k}\right|\right)^{p_{n}}<\infty\right\} .
$$

Also, some geometric properties of $V(\lambda, p)$ are studied in [9, 10] and [11].

Throughout this paper, the sequence $\left(p_{n}\right)$ is a bounded sequence of positive real numbers with

(b1) the sequence $\left(p_{n}\right)$ of positive real numbers is increasing and bounded with $\lim \sup p_{n}<\infty$ and $\liminf p_{n}>1$,

(b2) the sequence $\left(\lambda_{n}\right)$ is a nondecreasing sequence of positive real numbers tending to infinity, $\lambda_{0}=1$ and $\lambda_{n+1} \leq \lambda_{n}+1$ with $\sum_{n=0}^{\infty}\left(\frac{1}{\lambda_{n}}\right)^{p_{n}}<\infty$.

Also we define $e_{i}=(0,0, \ldots, 1,0,0, \ldots)$, where 1 appears at the $i$ th place for all $i \in \mathbb{N}$. 
Different classes of paranormed sequence spaces have been introduced and their different properties have been investigated. See [12-15] and [16].

For any bounded sequence of positive numbers $\left(p_{n}\right)$, we have the following well-known inequality $\left|a_{n}+b_{n}\right|^{p_{n}} \leq 2^{h-1}\left(\left|a_{n}\right|^{p_{n}}+\left|b_{n}\right|^{p_{n}}\right), h=\sup _{n} p_{n}$, and $p_{n} \geq 1$ for all $n \in \mathbb{N}$. See [17].

\section{Preliminary and notation}

Definition 2.1 A class of linear sequence spaces $E$ is called a special space of sequences (sss) having the following conditions:

(1) $E$ is a linear space and $e_{n} \in E$ for each $n \in \mathbb{N}$.

(2) If $x \in \omega, y \in E$ and $\left|x_{n}\right| \leq\left|y_{n}\right|$ for all $n \in \mathbb{N}$, then $x \in E$ 'i.e., $E$ is solid'

(3) If $\left(x_{n}\right)_{n=0}^{\infty} \in E$, then $\left(x_{\left[\frac{n}{2}\right]}\right)_{n=0}^{\infty}=\left(x_{0}, x_{0}, x_{1}, x_{1}, x_{2}, x_{2}, \ldots\right) \in E$, where $\left[\frac{n}{2}\right]$ denotes the integral part of $\frac{n}{2}$.

Example 2.2 $\ell_{p}$ is a special space of sequences for $0<p<\infty$.

Example 2.3 $\operatorname{ces}_{p}$ is a special space of sequences for $1<p<\infty$.

Definition 2.4 $U_{E}^{\mathrm{app}}:=\left\{U_{E}^{\mathrm{app}}(X, Y) ; X, Y\right.$ are Banach spaces $\}$, where $U_{E}^{\mathrm{app}}(X, Y):=\{T \in$ $\left.L(X, Y):\left(\alpha_{n}(T)\right)_{n=0}^{\infty} \in E\right\}$.

Theorem 2.5 $U_{E}^{\mathrm{app}}$ is an operator ideal if $E$ is a special space of sequences (sss).

Proof See [18].

We give here the sufficient conditions on the generalized de La Vallée Poussin's mean such that the class of all bounded linear operators between any arbitrary Banach spaces with $n$th approximation numbers of the bounded linear operators in the generalized de La Vallée Poussin's mean form an operator ideal.

\section{Main results}

Theorem 3.1 $U_{V(\lambda, p)}^{\mathrm{app}}$ is an operator ideal, if conditions (b1) and (b2) are satisfied.

Proof (1-i) Let $x, y \in V(\lambda, p)$ since

$$
\sum_{n=0}^{\infty}\left(\frac{1}{\lambda_{n}} \sum_{k \in I_{n}}\left|x_{k}+y_{k}\right|\right)^{p_{n}} \leq 2^{h-1}\left(\sum_{n=0}^{\infty}\left(\frac{1}{\lambda_{n}} \sum_{k \in I_{n}}\left|x_{k}\right|\right)^{p_{n}}+\sum_{n=0}^{\infty}\left(\frac{1}{\lambda_{n}} \sum_{k \in I_{n}}\left|y_{k}\right|\right)^{p_{n}}\right),
$$

$h=\sup _{n} p_{n}$, then $x+y \in V(\lambda, p)$.

(1-ii) Let $\lambda \in \mathbb{R}, x \in V(\lambda, p)$, then

$$
\sum_{n=0}^{\infty}\left(\frac{1}{\lambda_{n}} \sum_{k \in I_{n}}\left|\lambda x_{k}\right|\right)^{p_{n}} \leq \sup _{n}|\lambda|^{p_{n}} \sum_{n=0}^{\infty}\left(\frac{1}{\lambda_{n}} \sum_{k \in I_{n}}\left|x_{k}\right|\right)^{p_{n}}<\infty
$$

we get $\lambda x \in V(\lambda, p)$, from (1-i) and (1-ii), $V(\lambda, p)$ is a linear space.

To show that $e_{m} \in V(\lambda, p)$ for each $m \in \mathbb{N}$, since $\sum_{n=0}^{\infty}\left(\frac{1}{\lambda_{n}}\right)^{p_{n}}<\infty$. Thus we get

$$
\rho\left(e_{m}\right)=\sum_{n=m}^{\infty}\left(\frac{1}{\lambda_{n}} \sum_{k \in I_{n}}\left|e_{m}(k)\right|\right)^{p_{n}}=\sum_{n=m}^{\infty}\left(\frac{1}{\lambda_{n}}\right)^{p_{n}}<\infty
$$


Hence $e_{m} \in V(\lambda, p)$.

(2) Let $\left|x_{n}\right| \leq\left|y_{n}\right|$ for each $n \in \mathbb{N}$, then $\sum_{n=0}^{\infty}\left(\frac{1}{\lambda_{n}} \sum_{k \in I_{n}}\left|x_{k}\right|\right)^{p_{n}} \leq \sum_{n=0}^{\infty}\left(\frac{1}{\lambda_{n}} \sum_{k \in I_{n}}\left|y_{k}\right|\right)^{p_{n}}$ since $y \in V(\lambda, p)$. Thus $x \in V(\lambda, p)$.

(3) Let $\left(x_{n}\right) \in V(\lambda, p)$, then we have

$$
\begin{aligned}
\sum_{n=0}^{\infty} & \left(\frac{1}{\lambda_{n}} \sum_{k \in I_{n}}\left|x_{\left[\frac{k}{2}\right]}\right|\right)^{p_{n}} \\
= & \sum_{n=0}^{\infty}\left(\frac{1}{\lambda_{2 n}} \sum_{k \in I_{2 n}} \mid x_{\left[\frac{k}{2}\right]}\right)^{p_{2 n}}+\sum_{n=0}^{\infty}\left(\frac{1}{\lambda_{2 n+1}} \sum_{k \in I_{2 n+1}}\left|x_{\left[\frac{k}{2}\right]}\right|\right)^{p_{2 n+1}} \\
= & \sum_{n=0}^{\infty}\left(\frac{1}{\lambda_{2 n}}\left(\left(\sum_{k \in I_{n}} 2\left|x_{k}\right|\right)+\left|x_{n}\right|\right)\right)^{p_{n}}+\sum_{n=0}^{\infty}\left(\frac{1}{\lambda_{2 n+1}}\left(\sum_{k \in I_{n}} 2\left|x_{k}\right|\right)\right)^{p_{n}} \\
\leq & 2^{h-1}\left(\sum_{n=0}^{\infty}\left(\frac{1}{\lambda_{n}}\left(2 \sum_{k \in I_{n}}\left|x_{k}\right|\right)\right)^{p_{n}}+\sum_{n=0}^{\infty}\left(\frac{1}{\lambda_{n}} \sum_{k \in I_{n}}\left|x_{k}\right|\right)^{p_{n}}\right) \\
& +2^{h} \sum_{n=0}^{\infty}\left(\frac{1}{\lambda_{n}} \sum_{k \in I_{n}}\left|x_{k}\right|\right)^{p_{n}} \\
\leq & 2^{h-1}\left(2^{h}+1\right) \sum_{n=0}^{\infty}\left(\frac{1}{\lambda_{n}} \sum_{k \in I_{n}}\left|x_{k}\right|\right)^{p_{n}}+2^{h} \sum_{n=0}^{\infty}\left(\frac{1}{\lambda_{n}} \sum_{k \in I_{n}}\left|x_{k}\right|\right)^{p_{n}} \\
\leq & \left(2^{2 h-1}+2^{h-1}+2^{h}\right) \sum_{n=0}^{\infty}\left(\frac{1}{\lambda_{n}} \sum_{k \in I_{n}}\left|x_{k}\right|\right)^{p_{n}}<\infty .
\end{aligned}
$$

Hence $\left(x_{\left[\frac{n}{2}\right]}\right)_{n=0}^{\infty} \in V(\lambda, p)$. Hence from Theorem 2.5 it follows that $U_{V(\lambda, p)}^{\text {app }}$ is an operator ideal.

Corollary 3.2 $U_{\mathrm{ces}(p)}^{\mathrm{app}}$ is an operator ideal if $\left(p_{n}\right)$ is an increasing sequence of positive real numbers, $\lim _{n \rightarrow \infty} \sup p_{n}<\infty$ and $\lim _{n \rightarrow \infty} \inf p_{n}>1$.

Corollary 3.3 $U_{\mathrm{ces}_{p}}^{\mathrm{app}}$ is an operator ideal if $1<p<\infty$.

Theorem 3.4 The linear space $F(X, Y)$ is dense in $U_{V(\lambda, p)}^{\mathrm{app}}(X, Y)$ if conditions (b1) and (b2) are satisfied.

Proof First we prove that every finite mapping $T \in F(X, Y)$ belongs to $U_{V(\lambda, p)}^{\text {app }}(X, Y)$. Since $e_{m} \in V(\lambda, p)$ for each $m \in \mathbb{N}$ and $V(\lambda, p)$ is a linear space, then for every finite mapping $T \in$ $F(X, Y)$, i.e., the sequence $\left(\alpha_{n}(T)\right)_{n=0}^{\infty}$ contains only finitely many numbers different from zero. Now we prove that $U_{V(\lambda, p)}^{\text {app }}(X, Y) \subseteq \overline{F(X, Y)}$. Since letting $T \in U_{V(\lambda, p)}^{\text {app }}(X, Y)$ we get $\left(\alpha_{n}(T)\right)_{n=0}^{\infty} \in V(\lambda, p)$, and since $\rho\left(\left(\alpha_{n}(T)\right)_{n=0}^{\infty}\right)<\infty$, let $\left.\varepsilon \in\right] 0,1[$, then there exists a natural number $s>0$ such that $\rho\left(\left(\alpha_{n}(T)\right)_{n=s}^{\infty}\right)<\frac{\varepsilon}{2^{h+2} \delta c}$ for some $c \geq 1$, where $\delta=\max \left\{1, \sum_{n=s}^{\infty}\left(\frac{1}{\lambda_{n}}\right)^{p_{n}}\right\}$. 
Bakery Journal of Inequalities and Applications 2013, 2013:518

Page 5 of 8

Since $\alpha_{n}(T)$ is decreasing for each $n \in \mathbb{N}$, we get

$$
\begin{aligned}
\sum_{n=s+1}^{2 s}\left(\frac{1}{\lambda_{n}} \sum_{k \in I_{n}} \alpha_{2 s}(T)\right)^{p_{n}} & \leq \sum_{n=s+1}^{2 s}\left(\frac{1}{\lambda_{n}} \sum_{k \in I_{n}} \alpha_{n}(T)\right)^{p_{n}} \\
& \leq \sum_{n=s}^{\infty}\left(\frac{1}{\lambda_{n}} \sum_{k \in I_{n}} \alpha_{k}(T)\right)^{p_{n}}<\frac{\varepsilon}{2^{h+2} \delta c},
\end{aligned}
$$

then there exists $A \in F_{2 s}(X, Y), \operatorname{rank}(A) \leq 2 s$ with

$$
\sum_{n=2 s+1}^{3 s}\left(\frac{1}{\lambda_{n}} \sum_{k \in I_{n}}\|T-A\|\right)^{p_{n}} \leq \sum_{n=s+1}^{2 s}\left(\frac{1}{\lambda_{n}} \sum_{k \in I_{n}}\|T-A\|\right)^{p_{n}}<\frac{\varepsilon}{2^{h+2} \delta c},
$$

and since $\left(p_{n}\right)$ is a bounded sequence of positive real numbers, so we can take

$$
\sup _{n=s}^{\infty}\left(\sum_{k \in I_{s}}\|T-A\|\right)^{p_{n}}<\frac{\varepsilon}{2^{h} \delta}
$$

also $\alpha_{n}(T)=\inf \{\|T-A\|: A \in L(X, Y)$ and $\operatorname{rank}(A) \leq n\}$. Then there exists a natural mumberk $N>0, A_{N}$ with $\operatorname{rank}\left(A_{N}\right) \leq N$ and $\left\|T-A_{N}\right\| \leq 2 \alpha_{N}(T)$. Since $\alpha_{n}(T) \stackrel{n \rightarrow \infty}{\longrightarrow} 0$, then

$$
\left\|T-A_{N}\right\| \stackrel{N \rightarrow \infty}{\longrightarrow} 0, \quad \text { so we can take } \sum_{n=0}^{s}\left(\frac{1}{\lambda_{n}} \sum_{k \in I_{n}}\|T-A\|\right)^{p_{n}}<\frac{\varepsilon}{2^{h+3} \delta c}
$$

Since $\left(p_{n}\right)$ is an increasing sequence, by using (1), (2), (3) and (4), we get

$$
\begin{aligned}
d(T, A)= & \rho\left(\alpha_{n}(T-A)\right)_{n=0}^{\infty} \\
= & \sum_{n=0}^{3 s-1}\left(\frac{1}{\lambda_{n}} \sum_{k \in I_{n}} \alpha_{k}(T-A)\right)^{p_{n}}+\sum_{n=3 s}^{\infty}\left(\frac{1}{\lambda_{n}} \sum_{k \in I_{n}} \alpha_{k}(T-A)\right)^{p_{n}} \\
\leq & \sum_{n=0}^{3 s}\left(\frac{1}{\lambda_{n}} \sum_{k \in I_{n}}\|T-A\|\right)^{p_{n}}+\sum_{n=s}^{\infty}\left(\frac{1}{\lambda_{n}} \sum_{k \in I_{n+2 s}} \alpha_{k}(T-A)\right)^{p_{n+2 s}} \\
\leq & 3 \sum_{n=0}^{s}\left(\frac{1}{\lambda_{n}} \sum_{k \in I_{n}}\|T-A\|\right)^{p_{n}} \\
& +\sum_{n=s}^{\infty}\left(\frac{1}{\lambda_{n}} \sum_{k \in I_{2 s-1}} \alpha_{k}(T-A)+\frac{1}{\lambda_{n}} \sum_{k \in I_{n+2 s} I_{2 s-1}} \alpha_{k}(T-A)\right)^{p_{n}} \\
\leq & 3 \sum_{n=0}^{s}\left(\frac{1}{\lambda_{n}} \sum_{k \in I_{n}}\|T-A\|\right)^{p_{n}} \\
& +2^{h-1}\left(\sum_{n=s}^{\infty}\left(\frac{1}{\lambda_{n}} \sum_{k \in I_{2 s-1}} \alpha_{k}(T-A)\right)^{p_{n}}+\sum_{n=s}^{\infty}\left(\frac{1}{\lambda_{n}} \sum_{k \in I_{n+2 s} \backslash I_{2 s-1}} \alpha_{k}(T-A)\right)^{p_{n}}\right) \\
\leq & 3 \sum_{n=0}^{s}\left(\frac{1}{\lambda_{n}} \sum_{k \in I_{n}}\|T-A\|\right)^{p_{n}}
\end{aligned}
$$




$$
\begin{aligned}
& +2^{h-1}\left(\sum_{n=s}^{\infty}\left(\frac{1}{\lambda_{n}} \sum_{k \in I_{s}}\|T-A\|\right)^{p_{n}}+\sum_{n=s}^{\infty}\left(\frac{1}{\lambda_{n}} \sum_{k \in I_{n}} \alpha_{k+2 s}(T-A)\right)^{p_{n}}\right) \\
\leq & 3 \sum_{n=0}^{s}\left(\frac{1}{\lambda_{n}} \sum_{k=0}^{n}\|T-A\|\right)^{p_{n}} \\
& +2^{h-1} \sup _{n=s}^{\infty}\left(\sum_{k \in I_{s}}\|T-A\|\right)^{p_{n}} \sum_{n=s}^{\infty}\left(\frac{1}{\lambda_{n}}\right)^{p_{n}}+2^{h-1} \sum_{n=s}^{\infty}\left(\frac{1}{\lambda_{n}} \sum_{k \in I_{n}} \alpha_{k}(T)\right)^{p_{n}}<\varepsilon .
\end{aligned}
$$

Definition 3.5 A class of special space of sequences (sss) $E_{\rho}$ is called a pre-modular special space of sequences if there exists a function $\rho: E \rightarrow[0, \infty$ [ satisfying the following conditions:

(i) $\rho(x) \geq 0 \forall x \in E_{\rho}$ and $\rho(x)=0 \Leftrightarrow x=\theta$, where $\theta$ is the zero element of $E$,

(ii) there exists a constant $l \geq 1$ such that $\rho(\lambda x) \leq l|\lambda| \rho(x)$ for all values of $x \in E$ and for any scalar $\lambda$,

(iii) for some numbers $k \geq 1$, we have the inequality $\rho(x+y) \leq k(\rho(x)+\rho(y))$ for all $x, y \in E$,

(iv) if $\left|x_{n}\right| \leq\left|y_{n}\right|$ for all $n \in \mathbb{N}$, then $\rho\left(\left(x_{n}\right)\right) \leq \rho\left(\left(y_{n}\right)\right)$,

(v) for some numbers $k_{0} \geq 1$, we have the inequality $\rho\left(\left(x_{n}\right)\right) \leq \rho\left(\left(x_{\left[\frac{n}{2}\right]}\right)\right) \leq k_{0} \rho\left(\left(x_{n}\right)\right)$,

(vi) for each $x=(x(i))_{i=0}^{\infty} \in E$, there exists $s \in \mathbb{N}$ such that $\rho(x(i))_{i=s}^{\infty}<\infty$. This means the set of all finite sequences is $\rho$-dense in $E$,

(vii) for any $\lambda>0$, there exists a constant $\zeta>0$ such that $\rho(\lambda, 0,0,0, \ldots) \geq \zeta \lambda \rho(1,0,0,0, \ldots)$.

It is clear from condition (ii) that $\rho$ is continuous at $\theta$. The function $\rho$ defines a metrizable topology in $E$ endowed with this topology which is denoted by $E_{\rho}$.

Example 3.6 $\ell_{p}$ is a pre-modular special space of sequences for $0<p<\infty$, with $\rho(x)=$ $\sum_{n=0}^{\infty}\left|x_{n}\right|^{p}$.

Example 3.7 $\operatorname{ces}_{p}$ is a pre-modular special space of sequences for $1<p<\infty$, with $\rho(x)=$ $\sum_{n=0}^{\infty}\left(\frac{1}{n+1} \sum_{k=0}^{n}\left|x_{n}\right|\right)^{p}$.

Theorem 3.8 $V(\lambda, p)$ with $\rho(x)=\sum_{n=0}^{\infty}\left(\frac{1}{\lambda_{n}} \sum_{k \in I_{n}}\left|x_{n}\right|\right)^{p_{n}}$ is a pre-modular special space of sequences if conditions (b1) and (b2) are satisfied.

Proof (i) Clearly, $\rho(x) \geq 0$ and $\rho(x)=0 \Leftrightarrow x=\theta$.

(ii) Since $\left(p_{n}\right)$ is bounded, then there exists a constant $l \geq 1$ such that $\rho(\lambda x) \leq l|\lambda| \rho(x)$ for all values of $x \in E$ and for any scalar $\lambda$.

(iii) For some numbers $k=\max \left(1,2^{h-1}\right) \geq 1$, we have the inequality $\rho(x+y) \leq k(\rho(x)+$ $\rho(y))$ for all $x, y \in V(\lambda, p)$.

(iv) Let $\left|x_{n}\right| \leq\left|y_{n}\right|$ for all $n \in \mathbb{N}$, then $\sum_{n=0}^{\infty}\left(\frac{1}{\lambda_{n}} \sum_{k \in I_{n}}\left|x_{n}\right|\right)^{p_{n}} \leq \sum_{n=0}^{\infty}\left(\frac{1}{\lambda_{n}} \sum_{k \in I_{n}}\left|y_{n}\right|\right)^{p_{n}}$.

(v) There exist some numbers $k_{0}=2^{h-1}\left(2^{h}+1\right)+2^{h} \geq 1$; by using (iv) we have the inequality $\rho\left(\left(x_{n}\right)\right) \leq \rho\left(\left(x_{\left[\frac{n}{2}\right]}\right)\right) \leq k_{0} \rho\left(\left(x_{n}\right)\right)$.

(vi) It is clear that the set of all finite sequences is $\rho$-dense in $V(\lambda, p)$.

(vii) For any $\lambda>0$, there exists a constant $0<\zeta<\lambda^{p_{0}-1}$ such that $\rho(\lambda, 0,0,0, \ldots) \geq$ $\zeta \lambda \rho(1,0,0,0, \ldots)$. 
Theorem 3.9 Let $X$ be a normed space, $Y$ be a Banach space, and let conditions (b1) and (b2) be satisfied, then $U_{V_{\rho}(\lambda, p)}^{\mathrm{app}}(X, Y)$ is complete.

Proof Let $\left(T_{m}\right)$ be a Cauchy sequence in $U_{V_{\rho}(\lambda, p)}^{\mathrm{app}}(X, Y)$. Since $V(\lambda, p)$ with $\rho(x)=$ $\sum_{n=0}^{\infty}\left(\frac{1}{\lambda_{n}} \sum_{k \in I_{n}}\left|x_{n}\right|\right)^{p_{n}}$ is a pre-modular special space of sequences, then, by using condition (vii) and since $U_{V_{\rho}(\lambda, p)}^{\text {app }}(X, Y) \subseteq L(X, Y)$, we have $\rho\left(\left(\alpha_{n}\left(T_{i}-T_{j}\right)\right)_{n=0}^{\infty}\right) \geq \rho\left(\alpha_{0}\left(T_{i}-\right.\right.$ $\left.\left.T_{j}\right), 0,0,0, \ldots\right)=\rho\left(\left\|T_{i}-T_{j}\right\|, 0,0,0, \ldots\right) \geq \zeta\left\|T_{i}-T_{j}\right\| \rho(1,0,0,0, \ldots)$, then $\left(T_{m}\right)$ is also a Cauchy sequence in $L(X, Y)$. Since the space $L(X, Y)$ is a Banach space, then there exists $T \in L(X, Y)$ such that $\left\|T_{m}-T\right\| \stackrel{m \rightarrow \infty}{\longrightarrow} 0$ and since $\left(\alpha_{n}\left(T_{m}\right)\right)_{n=0}^{\infty} \in E$ for all $m \in \mathbb{N}, \rho$ is continuous at $\theta$ and using (iii), we have

$$
\begin{aligned}
\rho\left(\alpha_{n}(T)\right)_{n=0}^{\infty} & =\rho\left(\alpha_{n}\left(T-T_{m}+T_{m}\right)\right)_{n=0}^{\infty} \leq k \rho\left(\alpha_{\left[\frac{n}{2}\right]}\left(T_{m}-T\right)\right)_{n=0}^{\infty}+k \rho\left(\alpha_{\left[\frac{n}{2}\right]}\left(T_{m}\right)\right)_{n=0}^{\infty} \\
& \leq k \rho\left(\left(\left\|T_{m}-T\right\|\right)_{n=0}^{\infty}\right)+k \rho\left(\alpha_{n}\left(T_{m}\right)\right)_{n=0}^{\infty}<\varepsilon \quad \text { for some } k \geq 1 .
\end{aligned}
$$

Hence $\left(\alpha_{n}(T)\right)_{n=0}^{\infty} \in V_{\rho}(\lambda, p)$ as such $T \in U_{V_{\rho}}^{\mathrm{app}}(\lambda, p)(X, Y)$.

Corollary 3.10 Let $X$ be a normed space, $Y$ be a Banach space and $\left(p_{n}\right)$ be an increasing sequence of positive real numbers with $\lim \sup p_{n}<\infty$ and $\liminf p_{n}>1$, then $U_{\mathrm{ces}(p)}^{\mathrm{app}}(X, Y)$ is complete.

Corollary 3.11 Let $X$ be a normed space, $Y$ be a Banach space and $\left(p_{n}\right)$ be an increasing sequence of positive real numbers with $1<p<\infty$, then $U_{\operatorname{ces}_{p}}^{\mathrm{app}}(X, Y)$ is complete.

\section{Competing interests}

The author declares that he has no competing interests.

\section{Acknowledgements}

The author is most grateful to the editor and anonymous referee for careful reading of the paper and valuable suggestions which helped in improving an earlier version of this paper.

Received: 21 April 2013 Accepted: 9 September 2013 Published: 09 Nov 2013

\section{References}

1. Kalton, NJ: Spaces of compact operators. Math. Ann. 208, 267-278 (1974)

2. Lima, $\AA$, Oja, E: Ideals of finite rank operators, intersection properties of balls, and the approximation property. Stud. Math. 133, 175-186 (1999)

3. Pietsch, A: Operator Ideals. North-Holland, Amsterdam (1980)

4. Sanhan, W, Suantai, S: On k-nearly uniformly convex property in generalized Cesáro sequence space. Int. J. Math. Math. Sci. 57, 3599-3607 (2003)

5. Savas, E, Karakaya, V, Simşek, N: Some /(p)-type new sequence spaces and their geometric properties. Abstr. Appl. Anal. 2009, 69697 (2009)

6. Simşek, N, Karakaya, V: On some geometrical properties of generalized modular spaces of Cesáro type defined by weighted means. J. Inequal. Appl. 2009, Article ID 932734 (2009) 40605 (26E60)

7. Karakaya, V: Some geometric properties of sequence spaces involving lacunary sequence. J. Inequal. Appl. 2007, $81028(2007)$

8. Şimşek, N, Savas, E, Karakaya, V: Some geometric and topological properties of a new sequence space defined by de La Vallée Poussin mean. J. Comput. Anal. Appl. 12(4), 768-779 (2010)

9. Şimşek, N, Savas, E, Karakaya, V: On geometrical properties of some Banach spaces. Appl. Math. Inf. Sci. 7(1), 295-300 (2013)

10. Şimşek, N: On some geometric properties of sequence space defined by de La Vallée Poussin mean. J. Comput. Anal. Appl. 13(3), 565-573 (2011)

11. Çinar, M, Karakaş, M, Et, M: Some geometric properties of the metric space $V[\lambda, p]$. J. Inequal. Appl. 2013,28 (2013)

12. Rath, D, Tripathy, BC: Matrix maps on sequence spaces associated with sets of integers. Indian J. Pure Appl. Math. 27(2), 197-206 (1996)

13. Tripathy, BC, Sen, M: On generalized statistically convergent sequences. Indian J. Pure Appl. Math. 32(11), 1689-1694 (2001) 
14. Tripathy, BC, Chandra, P: On some generalized difference paranormed sequence spaces associated with multiplier sequences defined by modulus function. Anal. Theory Appl. 27(1), 21-27 (2011)

15. Tripathy, BC: Matrix transformations between some classes of sequences. J. Math. Anal. Appl. 206, 448-450 (1997)

16. Tripathy, BC: On generalized difference paranormed statistically convergent sequences. Indian J. Pure Appl. Math. 35(5), 655-663 (2004)

17. Altay, B, Başar, F: Generalization of the sequence space $\ell(p)$ derived by weighted means. J. Math. Anal. Appl. 330(1), 147-185 (2007)

18. Faried, N, Bakery, AA: Mappings of type Orlicz and generalized Cesáro sequence space. J. Inequal. Appl. (2013). doi:10.1186/1029-242X-2013-186

10.1186/1029-242X-2013-518

Cite this article as: Bakery: Mappings of type generalized de La Vallée Poussin's mean. Journal of Inequalities and Applications 2013, 2013:518

Submit your manuscript to a SpringerOpen ${ }^{\odot}$ journal and benefit from:

- Convenient online submission

- Rigorous peer review

- Immediate publication on acceptance

- Open access: articles freely available online

- High visibility within the field

- Retaining the copyright to your article

Submit your next manuscript at $>$ springeropen.com 